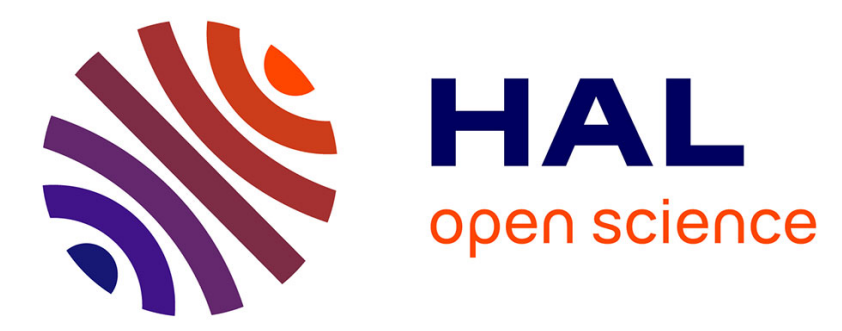

\title{
Asymmetrically Clipped-FSK Modulation for Energy Efficient Visible Light Communications
}

M. J. Khan, Ali Waqar Azim, Yannis Le Guennec, Ghislaine Maury, Laurent Ros

\section{- To cite this version:}

M. J. Khan, Ali Waqar Azim, Yannis Le Guennec, Ghislaine Maury, Laurent Ros. Asymmetrically Clipped-FSK Modulation for Energy Efficient Visible Light Communications. PIMRC 2021 - IEEE International Symposium on Personal, Indoor and Mobile Radio Communications (PIMRC 2021), Sep 2021, Oulu (Virtual conference), Finland. pp.458-464, 10.1109/PIMRC50174.2021.9569645 . hal03369258

\section{HAL Id: hal-03369258 \\ https://hal.science/hal-03369258}

Submitted on 7 Oct 2021

HAL is a multi-disciplinary open access archive for the deposit and dissemination of scientific research documents, whether they are published or not. The documents may come from teaching and research institutions in France or abroad, or from public or private research centers.
L'archive ouverte pluridisciplinaire HAL, est destinée au dépôt et à la diffusion de documents scientifiques de niveau recherche, publiés ou non, émanant des établissements d'enseignement et de recherche français ou étrangers, des laboratoires publics ou privés. 


\title{
Asymmetrically Clipped-FSK Modulation for Energy Efficient Visible Light Communications
}

\author{
Muhammad Jehangir Khan*, Ali Waqar Azim ${ }^{\dagger}$, Yannis Le Guennec*, Ghislaine Maury ${ }^{\ddagger}$, Laurent Ros* \\ ${ }^{*}$ Univ. Grenoble Alpes, CNRS, Grenoble INP, GIPSA-Lab, 38000 Grenoble, France \\ \{muhammad-jehangir.khan,yannis.leguennec,laurent.ros\}@gipsa-lab.grenoble-inp.fr \\ $\dagger$ Telecommunication Engineering Department, University of Engineering and Technology, Taxila, 47050, Pakistan \\ aliwaqarazim@gmail.com \\ ${ }_{\ddagger}^{\ddagger}$ Univ. Grenoble Alpes, CNRS, Grenoble INP, IMEP-LAHC, 38000 Grenoble, France \\ maury@grenoble-inp.fr
}

\begin{abstract}
In this article, we present proof-of-concept of $M$ ary asymmetrically clipped (AC)-frequency-shift keying (FSK) for energy-efficient/low data rate visible light communications (VLC). $M$-ary AC-FSK uses the odd frequency waveforms from $2 M$-ary FSK; which exhibit half-wave symmetry. Owing to this half-wave symmetry, the negative amplitude excursions are clipped without loss of information. Hereby, we investigate the performance of three different receivers for $M$-ary AC-FSK exhibiting different complexities. These receivers are: (i) an optimal time-domain maximum likelihood (ML) receiver; (ii) 1tap discrete cosine transform (DCT) receiver; and (iii) a 2-tap harmonic receiver which exploits the spectral properties of $\mathrm{AC}$ FSK waveforms. We shall exemplify that the 2-tap harmonic receiver overcomes the energy efficiency versus complexity trade-off that exists for optimal ML and 1-tap DCT receivers. Simulation results shall confirm that the performance of 2-tap harmonic receiver reaches the performance of the previously proposed unipolar (U)-FSK with the optimal receiver, but with a drastically reduced receiver complexity.
\end{abstract}

Index Terms-Frequency-shift keying, visible light communication, intensity-modulation and direct-detection, Internet-ofThings.

\section{INTRODUCTION}

W ith the advent of Internet-of-Everything (IoE), there is a growing demand for wireless connectivity leading to radio-frequency (RF) spectrum saturation. In this scenario, Visible Light Communications (VLC) offer considerable interest by providing a large unlicensed spectrum for wireless communications without any interference with the RF spectrum [1]. VLC could rely on the massive deployment of light emitting diodes (LEDs) for lighting applications and use simple intensity-modulation and direct-detection (IM-DD) [2] In the perspective of $\mathrm{IoE}$, a large number of communications will deal with the limited amount of data, without high data rate constraints. Thus, VLC systems dedicated to these low data rate communications should be energy-efficient to extend their communication range and/or limit their power consumption.

To define an energy-efficient VLC system dedicated to low data rate communications, the choice of digital modulation is of fundamental importance. Due to the IM-DD constraint, the modulation signal must be unipolar and realvalued [1]. Among available modulation schemes, two groups are specially identified: (i) the linear modulations; and (ii) the orthogonal modulations. On one hand, linear modulation and their multicarrier extensions have been extensively studied for VLC, including $M$-ary pulse amplitude modulation (PAM) [3] and optical-orthogonal frequency-division multiplexing (OOFDM) [4]. These modulation schemes can reach high spectral efficiency by increasing modulation order $M$ but at the expense of low energy efficiency (i.e., the necessary electrical signal-to-noise ratio (SNR) per bit to achieve a target bit error rate (BER) increases). On the other hand, orthogonal modulations, such as pulse position modulation (PPM) or frequency-shift keying (FSK), are known in RF domain to show higher energy efficiency than linear modulations, at the expense of degraded spectral efficiencies [5]. Recently, FSKbased modulations have raised interest for VLC applications, due to their high energy efficiency, quasi constant envelop property to circumvent LED non-linearity and easy channel equalization. As conventional $M$-ary FSK is not directly compatible with IM-DD because of its bipolar nature, two variants have been introduced in [6]: $M$-ary direct-current (DC)-FSK and $M$-ary unipolar (U)-FSK. $M$-ary DC-FSK is derived from bipolar $M$-ary FSK by adding a DC-offset to make the waveform positive. Nevertheless, the added DCoffset leads to a large waste of energy. $M$-ary U-FSK has been introduced to avoid the introduction of this DC-offset: it sequentially transmits the positive samples of the FSK waveform during the first symbol period and the sign-flipped negative samples during a consecutive symbol period; a similar approach for OFDM-based VLC is reported in [7]. Simulation results presented in [6] confirmed that $M$-ary U-FSK reaches a large energy efficiency gain over both DC-FSK and linear modulations, especially when optimal maximum likelihood ML receiver is used. Nevertheless, the complexity of the optimal ML receiver is high, consequently, only degraded energy efficiency is obtained when a low complexity suboptimal reconstruction receiver is considered [6].

In this paper, we propose a new variant of $M$-ary FSK called $M$-ary Asymmetrically Clipped (AC)-FSK that is compatible with IM-DD VLC systems. $M$-ary AC-FSK benefits from its waveform spectral properties to build a low complexity frequency-domain harmonic receiver, i.e., the 2-tap harmonic 
receiver that reaches the performance of the optimal ML receiver. It shall be demonstrated that the energy efficiency obtained via the 2-tap harmonic receiver for AC-FSK is close to that of the optimal ML receiver of U-FSK, however, with significantly reduced complexity.

Unless otherwise mentioned, we use lower-case boldface italic letters to represent discrete time-domain vectors, e.g., $\boldsymbol{s}_{m}$, where $m$ in the subscript represents the activated frequency. The $n$th sample of discrete time-domain waveform is given as $\boldsymbol{s}_{m}[n]$, while its analog domain counterpart is represented as $\boldsymbol{s}_{m}(t)$. The upper-case boldface italic letters are used to represent the frequency-domain counterparts of the discrete time-domain waveforms, e.g., $\boldsymbol{S}_{m}$, for which the frequency component at $k$ th frequency is given as $S_{m}[k]$. Matrices are denoted by boldface caligraphic letters, e.g., $\mathcal{D}$. The operations such as transpose, inner product, absolute value and Euclidean norm are respectively represented by $(\cdot)^{\mathrm{T}},\langle\cdot, \cdot\rangle$, $|\cdot|$ and $\|\cdot\|$. Moreover, the discrete cosine transform (DCT) matrix of size $M$ is represented as $\mathcal{C}_{M}$ whose $\left(n_{1}, n_{2}\right)$ th element is given as [8]:

$\mathcal{C}_{M}\left(n_{1}, n_{2}\right)=\left\{\begin{array}{ll}\frac{1}{\sqrt{M}} & n_{1}=0 \\ \sqrt{\frac{2}{M}} \cos \left(\frac{\pi\left(2 n_{2}+1\right) n_{1}}{2 M}\right) & 1 \leq n_{1} \leq M-1\end{array}\right.$.

where $0 \leq n_{2} \leq M-1$.

The rest of the paper is organized as follows. In section II, $M$-ary U-FSK and its conventional receivers are revisited. In section III, we present $M$-ary AC-FSK along with its transmitter and receiver architectures, with the perspective of defining a low complexity harmonic receiver able to reach the optimal ML receiver performances. In section IV, simulation results such as BER performance in AWGN channel, energy efficiency versus spectral efficiency and complexity comparisons are presented to highlight the benefit of our proposed AC-FSK modulation scheme over U-FSK technique. Finally, conclusions and perspectives are drawn in section $\mathrm{V}$.

\section{PRELIMINARIES}

As the simulation results in [6] confirmed that $M$-ary UFSK is more energy-efficient than $M$-ary DC-FSK, here we revisit only the $M$-ary U-FSK for reference purpose. However, firstly, we present the system model.

\section{A. System Model}

Considering an additive white Gaussian noise (AWGN) VLC channel, the received waveform $r(t)$ is contaminated by ambient noise, $n(t)$. After impinging $r(t)$ on an analog-todigital converter (ADC), we obtain:

$$
\boldsymbol{r}=\boldsymbol{s}_{m}+\boldsymbol{w},
$$

where $\boldsymbol{w}$ is the AWGN having mono-lateral power spectral density (PSD) of $N_{0}$, i.e., $\boldsymbol{w}=$ $\left[w[0], \cdots, w\left[M_{\mathrm{c}}-1\right]\right]^{\mathrm{T}}, \boldsymbol{s}_{\boldsymbol{m}}=\left[s_{m}[0], \cdots, s_{m}\left[M_{\mathrm{c}}-1\right]\right]^{\mathrm{T}}$ and $\boldsymbol{r}=\left[r[0], \cdots, r\left[M_{\mathrm{c}}-1\right]\right]^{\mathrm{T}}$.

\section{B. M-ary U-FSK Signaling}

The $m$ th frequency waveform $\tilde{s}_{m}$, of the conventional bipolar $M$-ary FSK dictionary, $\mathcal{D}^{M-F S K}$ is given as [6]:

$$
\tilde{\boldsymbol{s}}_{m}=\mathcal{C}_{M}^{\mathrm{T}} \boldsymbol{F}_{m},
$$

where $\boldsymbol{F}_{m}=[0, \cdots, 1, \cdots, 0]^{\mathrm{T}}$ specifies the activated frequency, e.g., the position of 1 in the vector identify the active frequency.

Time-domain $M$-ary FSK waveforms are in the form $\tilde{s}_{m}(t)=A \cos (2 \pi m \tilde{\Delta} f t)$, where $\tilde{\Delta f}=1 / 2 \tilde{T}_{s}$ is the frequency spacing between two waveforms, which is the minimal value that ensures orthogonality and $\tilde{T}_{s}$ is the symbol time. The bandwidth of the $M$-ary FSK signal is $\tilde{B} \approx M \tilde{\Delta f}$. All $M$-ary FSK symbols have the same energy, i.e., $E_{s}^{M-F S K}=$ $\left\|\tilde{\boldsymbol{s}}_{m}\right\|^{2}$.

U-FSK signaling creates real-valued and unipolar waveforms from the conventional bipolar $M$-ary FSK alphabet. The positive and negative amplitudes of $\tilde{s}_{m}[n]$ are obtained as [6]:

$$
\begin{aligned}
& \tilde{s}_{m}^{+}[n]=\frac{1}{2}\left(\tilde{s}_{m}[n]+\left|\tilde{s}_{m}[n]\right|\right), \\
& \tilde{s}_{m}^{-}[n]=\frac{1}{2}\left(\tilde{s}_{m}[n]-\left|\tilde{s}_{m}[n]\right|\right) .
\end{aligned}
$$

Afterwards, the negative part $\tilde{s}_{m}^{-}[n]$ of each conventional bipolar $M$-ary FSK waveform is flipped and is transmitted in a consecutive symbol period, while the original negative samples are set to zero. This leads to the generation of $M$-ary U-FSK dictionary $\mathcal{D}^{U}$. For $M$-ary U-FSK, the symbol time $T_{s}$ and number of chips per symbol $M_{c}$ become double as compared to $M$-ary FSK symbol time $\tilde{T}_{s}$ and number of chips per symbol $M$, i.e., $T_{s}=2 \tilde{T}_{s}$ and $M_{c}=2 M$, respectively. For $M$-ary U-FSK, DC waveform $s_{0}$ is not used for transmission since ambient light may strongly corrupt it [7].

Fig. 1 (a) and (c) illustrate the time-domain $M$-ary UFSK waveforms for index $m=1$ and 2 along with their frequency-domain counterparts in (b) and (d), respectively, that are obtained through DCT, considering a modulation order $M=16$. From spectra shown in Fig. 1 (b), we can notice that most of the energy for odd frequency waveform $(m=1)$ is contained in 2 significant taps (marked as "1" and "2", ignoring the DC tap), while the other harmonics have relatively smaller amplitude (marked as "4" and "6"). For even frequency waveform $(m=2)$, it can be seen from Fig. 1 (d) that the energy is contained in a large number of non-negligible taps. Therefore, we need to consider more non-negligible taps for even frequency waveforms.

\section{M-ary U-FSK Conventional Receivers}

1) Optimal ML time-domain receiver: The theoretically optimal ML time-domain (TD) receiver is presented in [6] for $M$-ary U-FSK . It permits to guarantee a minimum error probability in case of independent and equiprobable symbols. Under that condition, optimal ML TD receiver relies on evaluating the correlation of the received waveform $r$ with all 

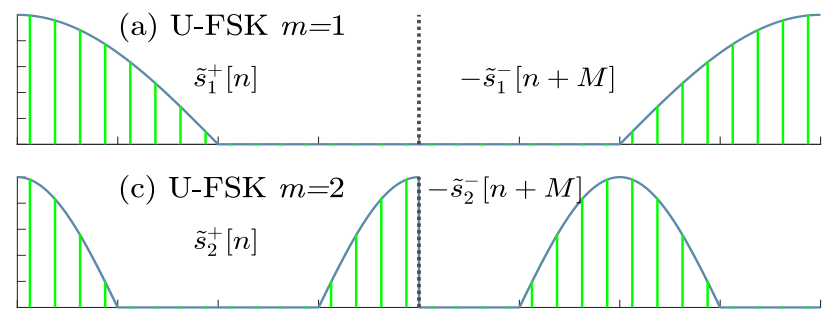

(e) AC-FSK $m=1$
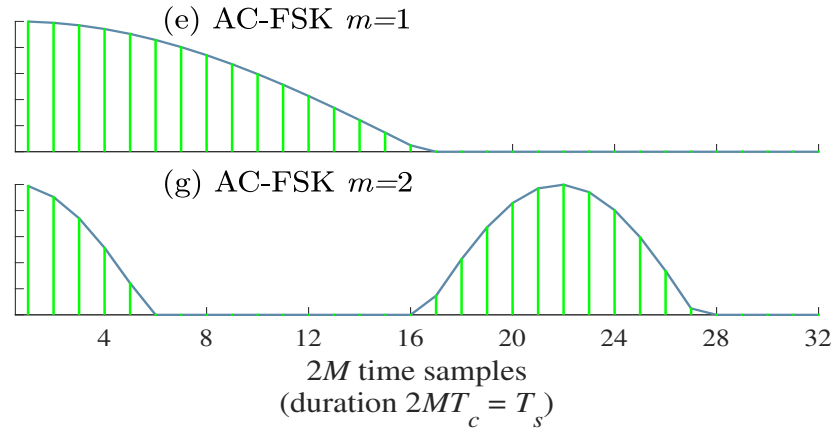

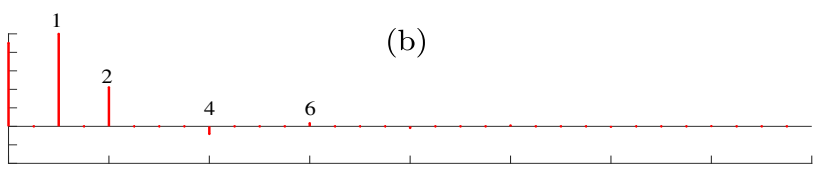

(d)

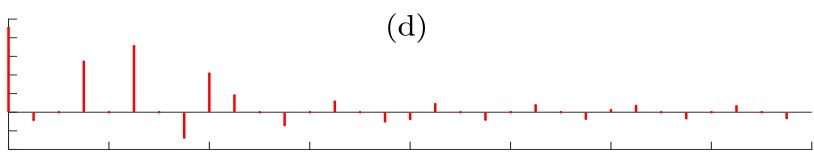

(f)

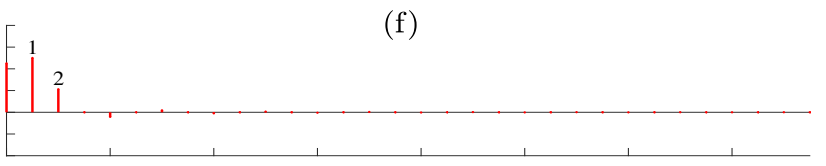

(h)

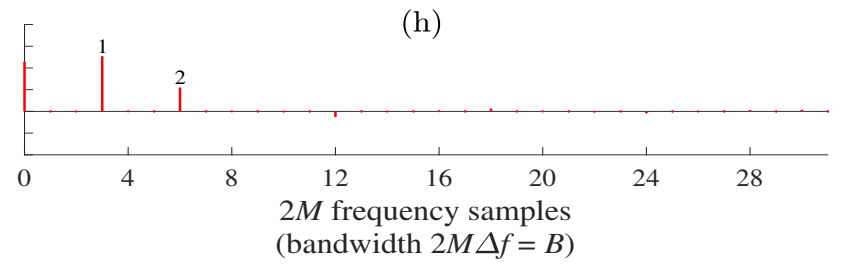

Fig. 1: (a) and (c) represent time-domain $M$-ary U-FSK odd and even waveforms ( $m=1,2$ respectively), while (b) and (d) represent their counterpart in frequency-domain, considering $M=16$. (e) and (g) represent time-domain $M$-ary AC-FSK waveforms ( $m=1,2$ respectively), while (f) and (h) represent their counterpart in frequency-domain, considering $M=16$.

the dictionary waveforms. The waveform index $\hat{m}$ is obtained as:

$$
\hat{m}=\underset{m}{\arg \max }\left\{\left\langle\boldsymbol{r}, \boldsymbol{s}_{m}\right\rangle\right\} .
$$

It should be noticed that the optimal ML TD receiver has high complexity, where the number of (non-zero) real multiplications is of $\mathcal{O}\left(M^{2}\right)$.

2) Sub-optimal Reconstruction receiver: The reconstruction receiver is a sub-optimal DCT-based receiver and its structure for $M$-ary U-FSK is presented in [6]. The process for identifying the waveform index consists in rebuilding original FSK (noisy) waveforms, $\tilde{r}[n]$ for $n=0,1, \cdots, M-1$ from $r[\bar{n}]$ for $\bar{n}=0,1, \cdots, 2 M-1$ as:

$$
\tilde{r}[n]=r[n]-r[M+n] .
$$

Subsequently, $\boldsymbol{R}=\left[R_{0}, R_{1}, \cdots, R_{M-1}\right]^{\mathrm{T}} \in \Re^{M}$ is obtained from $\tilde{r}[n]$ by using $M$-order DCT. Thereafter, the index $\hat{m}$ of the waveform is identified as:

$$
\hat{m}=\underset{m}{\arg \max }\left\{\left|R_{m}\right|\right\} .
$$

The reconstruction-based receiver exhibits low complexity compared to the optimal ML TD receiver as the real multiplications are of $\mathcal{O}\left(M \log _{2}(M) / 2\right)$ due to the $M$-order DCT [9]. Nevertheless, the reconstruction receiver induces a significant energy efficiency penalty (about $1 \mathrm{~dB}$ [6]) compared to the optimal time-domain receiver. Indeed, U-FSK symbol reconstruction subtracts noisy samples to retrieve the original FSK waveform. This process restores the orthogonality of the FSK waveforms but at the price of doubling the noise variance, considering the same symbol energy.

\section{Proposed $M$-ARY AC-FSK Modulation}

In this section, we present $M$-ary AC-FSK which is also compatible with IM-DD VLC systems. We shall demonstrate that $M$-ary AC-FSK can achieve a similar performance as UFSK optimal ML receiver, but with a significantly reduced receiver complexity.

\section{A. M-ary AC-FSK Principle}

$M$-ary AC-FSK is derived from Asymmetrically Clipped OFast-OFDM (ACO-FOFDM) that has been extensively studied for high data rate VLC systems [9]. In ACO-OFDM, only odd frequency waveforms are selected because they exhibit a socalled half-wave symmetry property, i.e., for each sample $x[n]$ in the positive part of the time-domain waveform, there exists a negative sample with the same amplitude in the negative part of the waveform, such as [9]:

$$
x[n]=-x[N-1-n], \quad 0 \leq n \leq N / 2-1 .
$$

As a consequence of the half-wave symmetry, the negative part of the odd frequency waveforms can be clipped to zero without any loss of information. Additionally, subcarrier harmonics generated by clipping do not interfere with data carriers, since harmonic frequencies of odd frequency subcarriers only fall into even frequency subcarriers.

The proposed $M$-ary AC-FSK modulation technique relies also on the use of odd frequency waveforms by taking benefit from half-wave symbol symmetry and related spectral consequences. Contrary to ACO-FOFDM, there is no potential interference issue to consider with other symbol carriers, since only a single frequency is active for AC-FSK symbol per symbol period. 


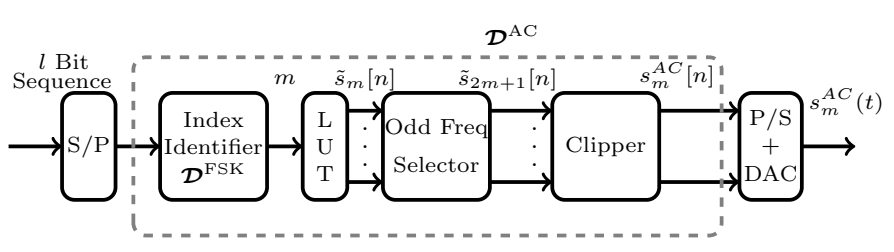

Fig. 2: Transmitter configuration of $M$-ary AC-FSK

\section{B. $M$-ary AC-FSK Signaling}

$M$-ary AC-FSK transmitter structure is illustrated in Fig. 2. Initially, the odd frequency waveforms, $\tilde{s}_{\tilde{m}}[n]$ with $\tilde{m} \in$ $\{1,3, \ldots, 2 M-3,2 M-1\}$, are selected from conventional bipolar $2 M$-ary FSK dictionary $\mathcal{D}^{2 M-F S K}$. Thereafter, the $M$-ary AC-FSK waveform, $s_{m}^{A C}[n]$ where $0 \leq n \leq 2 M-1$ corresponding to the $m$ th frequency is extracted by clipping to zero the negative samples of $\tilde{s}_{\tilde{m}}[n], \tilde{m} \in\{1,3, \ldots, 2 M-$ $3,2 M-1\}$ as:

$$
s_{m}^{A C}[n]=\tilde{s}_{\tilde{m}}^{+}[n], \quad \text { with } \quad m=(\tilde{m}+1) / 2,
$$

where $\tilde{s}_{\tilde{m}}^{+}[n]$ represents the positive amplitudes of conventional bipolar $2 M$-ary FSK odd frequency waveforms while the negative samples are clipped to zero. Fig. 1 (e) and (g) represent the time-domain AC-FSK waveforms (for $m=1$ and 2 respectively). Fig. 1 (f) and (h) represent the frequency-domain AC-FSK waveforms, $\boldsymbol{S}_{m}$ after $2 M$-order DCT (for $m=1$ and 2 respectively). As it can be seen from Fig. 1 (f) and (h), only 2 significant harmonics (leaving aside the DC component) are present (marked as "1" and " 2 ") in the waveform spectrum that contains most of the energy.

The cardinality of the AC-FSK dictionary, $\mathcal{D}^{A C}$ is half of $2 M$-ary FSK dictionary, $\mathcal{D}^{2 M-F S K}$. For an identical chip duration $T_{c}, M$-ary AC-FSK symbols have a symbol period $T_{s}$ which is the same as the $2 M$-ary FSK symbol period. The analog domain $m$ th waveform of $M$-ary AC-FSK, $s_{m}^{\mathrm{AC}}(t)$ is obtained from (10) after digital to analog conversion (DAC) using $t=\left(n+\frac{1}{2}\right) T_{c}$. The electrical power of $M$-ary AC-FSK is half of the electrical power of bipolar $M$-ary FSK, and is equal to $A^{2} / 4$, as for $M$-ary U-FSK. All the $M$-ary AC-FSK symbols have the same electrical energy. Then, the average electrical energy per symbol for $M$-ary AC-FSK, $E_{\mathrm{s}_{(\text {elec })}}^{\mathrm{AC}}$ is expressed as:

$$
E_{\mathrm{s}_{(\text {elec })}}^{\mathrm{AC}}=\int_{0}^{T_{\mathrm{s}}}\left|s_{m}^{\mathrm{AC}}(t)\right|^{2} \mathrm{~d} t=\frac{A^{2}}{4} T_{s} .
$$

TABLE I: Quantitative comparison of spectral efficiencies of conventional bipolar $M$-ary FSK, $M$-ary DC-FSK, $M$-ary U-FSK and $M$-ary AC-FSK for the same modulation order $M$.

\begin{tabular}{lc}
\hline Modulation Scheme & $\eta$ \\
\hline FSK & $\frac{2 \log _{2}(M)}{M}$ \\
DC-FSK & $\frac{2 \log _{2}(M)}{M}$ \\
U-FSK & $\frac{\log _{2}(M)}{M}$ \\
AC-FSK & $\frac{\log _{2}(M)}{M}$ \\
\hline
\end{tabular}

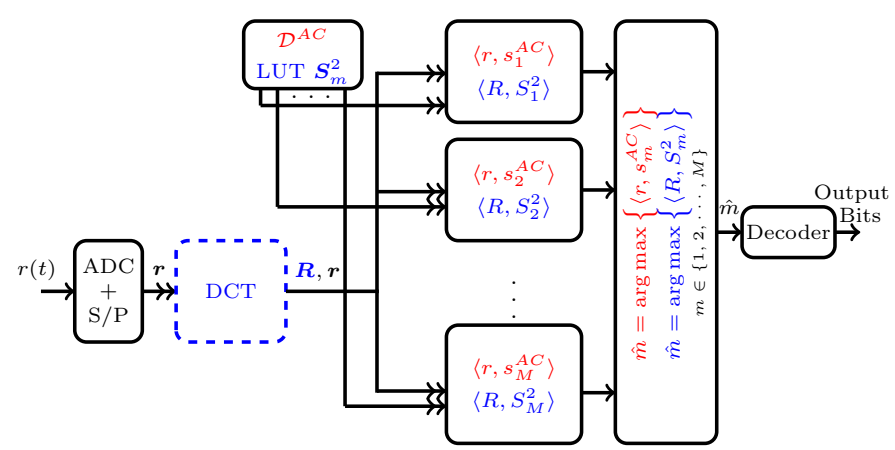

Fig. 3: Optimal ML TD receiver (red/black) and 2-tap harmonic receiver (blue/black) structure for $M$-ary AC-FSK.

\section{Spectral Efficiency}

The spectral efficiency of $M$-ary AC-FSK is expressed as:

$$
\eta^{A C}=\frac{R_{b}^{A C}}{B^{A C}}=\frac{\log _{2}(M)}{M} .
$$

where $R_{b}^{A C}$ is the bit rate, i.e., $R_{b}^{A C}=\log _{2}(M) / T_{s}$ and $B^{A C}$ is the bandwidth for $M$-ary AC-FSK, i.e., $B^{A C} \approx M \Delta f=\frac{M}{T_{s}}$ for sufficiently large $M$ (neglecting out-of-band energy on the harmonics of clipped waveform for $M$-ary AC-FSK). Table I summarizes the spectral efficiencies of conventional bipolar $M$-ary FSK, $M$-ary DC-FSK, $M$-ary U-FSK and $M$-ary AC-FSK for the same modulation order $M$. We can noticed that $M$-ary AC-FSK and $M$-ary U-FSK have identical spectral efficiencies but $M$-ary DC-FSK exhibits a double spectral efficiency for the same modulation order $M$, i.e., $\eta^{A C}=\eta^{U}=\eta^{D C} / 2$.

\section{M-ary AC-FSK Receivers}

1) Optimal time-domain Receiver: The optimal ML TD receiver for $M$-ary AC-FSK is illustrated in Fig. 3 with (red/black). The received waveform $r(t)$ is converted into digital domain, i.e., $r[n]$ for $n=0,1, \ldots, 2 M-1$ by using analog-to-digital converter. As all the waveforms in the dictionary, $\mathcal{D}^{A C}$ are of equal energy, so the transmit frequency is identified via the cross correlation in TD of the $r$ and $\boldsymbol{s}_{m}^{A C} \in \mathcal{D}^{A C}$ as:

$$
\hat{m}=\underset{m}{\arg \max }\left\langle\boldsymbol{r}, \boldsymbol{s}_{\boldsymbol{m}}^{\boldsymbol{A C}}\right\rangle \quad m \in\{1,2, \ldots, M\} .
$$

It is recalled that the number of chips per symbol $M_{c}$ for $M$-ary AC-FSK is doubled as compared to $M$-ary FSK, i.e., $M_{c}=2 M$ and half of the chips correspond to zeros. Thus, the complexity (in terms of real multiplication) of the optimal ML TD receiver for $M$-ary AC-FSK is of $\mathcal{O}\left(M^{2}\right)$, i.e., same as the complexity of U-FSK optimal ML receiver.

2) 1-tap DCT Receiver: It is a conventional sub-optimal DCT-based receiver typically used for ACO-FOFDM [9]. The 1-tap DCT receiver architecture is shown in Fig. 4. On the digitized received signal, $r[n]$ where $n=0,1, \cdots, 2 M-1$, a $2 M$-order DCT is applied, as in (1), resulting in $\boldsymbol{R}$ as:

$$
\boldsymbol{R}=\mathcal{C}_{2 M} \boldsymbol{r},
$$




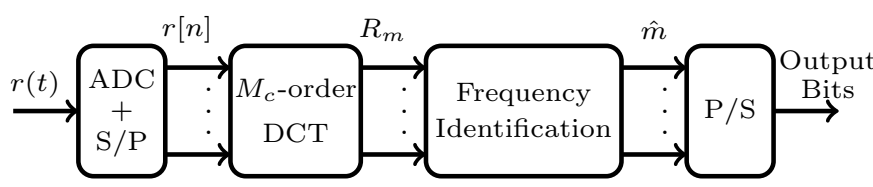

Fig. 4: Sub-optimal 1-tap DCT receiver structure for $M$-ary AC-FSK

where $\boldsymbol{R}=\left[R_{0}, R_{1}, \cdots, R_{2 M-1}\right]^{\mathrm{T}}$ and $\mathcal{C}_{2 M}$ is the $2 M$-order DCT matrix. Thereafter, the frequency index $m$ is identified from $\boldsymbol{R}$ as:

$$
\hat{m}=\underset{m}{\arg \max }\left\{\left|R_{2 m-1}\right|\right\} \quad m \in\{1,2, \ldots, M\} .
$$

The 1-tap DCT receiver has degraded performance compared to the optimal receiver due to the fact that only the (odd) tap with maximum amplitude is used for frequency index identification. Nevertheless, the 1-tap DCT receiver has a low complexity (in terms of real multiplications) is of $\mathcal{O}\left(M \log _{2}(2 M)\right)$, due to the $2 M$-order DCT [9].

From the 2 previous subsections (III.D.1) and (III.D.2), we conclude that there exists an energy efficiency vs complexity trade-off for optimal ML TD and 1-tap DCT receivers.

3) 2-tap Harmonic Receiver: The 2-tap harmonic receiver architecture for $M$-ary AC-FSK is illustrated in Fig. 3 with (blue/black). It uses a $M_{c}$-order DCT together with frequencydomain cross-correlation process to recover the transmitted symbols. In a first off-line step, DCT is applied to all the $M$-ary AC-FSK waveforms in the dictionary, $\mathcal{D}^{A C}$. Then, the two most significant taps selected from each $2 M$-ary AC-FSK frequency-domain waveform to build $\boldsymbol{S}_{m}^{2}$, leaving aside the DC tap (see spectra in Fig. 1 (f) and (h)) are stored in a LUT. These two significant taps (actually one odd, and one even) contain most of the symbol energy. On the digitized received signal, $r[n]$ where $n=0,1, \cdots, 2 M-1$, a $2 M$-order DCT is applied to obtain $\boldsymbol{R}$ as in (14). So, the on-line front-end processing is the same as for the the 1-tap DCT receiver, but the transmitted frequency is then identified by looking for a maximum after 2 taps-based computation (instead of 1 tap) in ouptut of the DCT (frequency-domain cross-correlation between $\boldsymbol{R}$ and the the 2 taps frequency-domain waveforms $\boldsymbol{S}_{m}^{2}$ in the LUT) as:

$$
\hat{m}=\underset{m}{\arg \max }\left\langle\boldsymbol{R}, \boldsymbol{S}_{m}^{2}\right\rangle .
$$

The 2-tap harmonic receiver is a low complexity receiver (in terms of real multiplications) is of $\mathcal{O}\left(M \log _{2}(2 M)+2 M\right)$ for $M$-ary AC-FSK. The 2-tap harmonic receiver can overcome the energy efficiency vs complexity trade-off found for optimal versus sub-optimal receivers.

It can be noticed that harmonic receiver could be applied as well to U-FSK receiver, but the number of taps needed for even frequency waveforms would be definitely larger than 2 (see Fig. 1. (d)). As a consequence, the harmonic receiver would be of higher complexity for U-FSK than the 2-tap harmonic receiver implemented for AC-FSK.

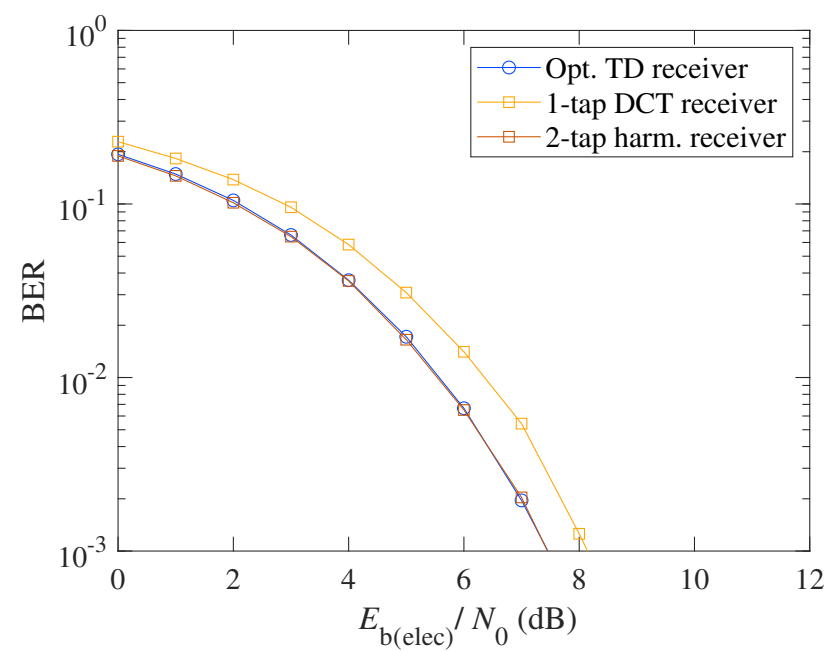

Fig. 5: BER vs $E_{b(e l e c)} / N_{0}$ comparison between optimal time-domain receiver, sub-optimal receiver and 2-tap harmonic receiver for $M$-ary ACFSK for modulation orders $\mathcal{D}=16$

\section{Simulation Results}

In this section, we have presented simulation results of already presented two variants of FSK based modulation schemes, i.e., $M$-ary DC-FSK and $M$-ary U-FSK, along with the new approach, i.e., $M$-ary AC-FSK. We evaluate the following performance parameters for $M$-ary AC-FSK: (i) BER performance comparison between receivers; (ii) BER as a function of SNR per bit considering an AWGN channel with optimal receiver; and (iii) complexity comparison between receivers. Monte Carlo simulation results are averaged over $10^{6}$ separate runs for AWGN channel.

\section{A. BER Performance Comparison between Receivers}

The BER performance comparison as a function of electrical signal-to-noise ratio per bit $E_{b(e l e c)} / N_{0}$ for optimal ML TD receiver, 1-tap DCT receiver and 2-tap harmonic receiver for $M$-ary AC-FSK, considering modulation order $\mathcal{D}^{A C}=16$ is illustrated in Fig. 5. As expected, the BER performance of optimal ML TD receiver is better than sub-optimal receiver over AWGN channel by less than 1dB. In Fig. 5, it can also be seen that the 2-tap harmonic receiver shows exactly the same BER performance as the optimal time-domain receiver.

\section{B. BER performance over AWGN with optimal receiver}

Fig. 6 depicts the spectral efficiency $\eta$ with respect to the required $E_{b(\text { elec })} / N_{0}$ to target a BER of $10^{-3}$ for $M$-ary ACFSK, $M$-ary U-FSK and $M$-ary DC-FSK. From Fig. 6, it can be seen that $M$-ary AC-FSK has far better performance than $M$-ary DC-FSK because the waveforms in the dictionary, $\mathcal{D}^{D C}$ are negatively affected due to DC-offset. Moreover $M$ ary AC-FSK also has slightly better performance than $M$ ary U-FSK in terms of spectral efficiency $\eta$ versus required $E_{b(e l e c)} / N_{0}$. We can see from Fig. 6 that for any targeted spectral efficiency $\eta$, we need about $0.1 \mathrm{~dB}$ less $E_{b(e l e c)} / N_{0}$ for $M$-ary AC-FSK as compared to $M$-ary U-FSK. Moreover, 


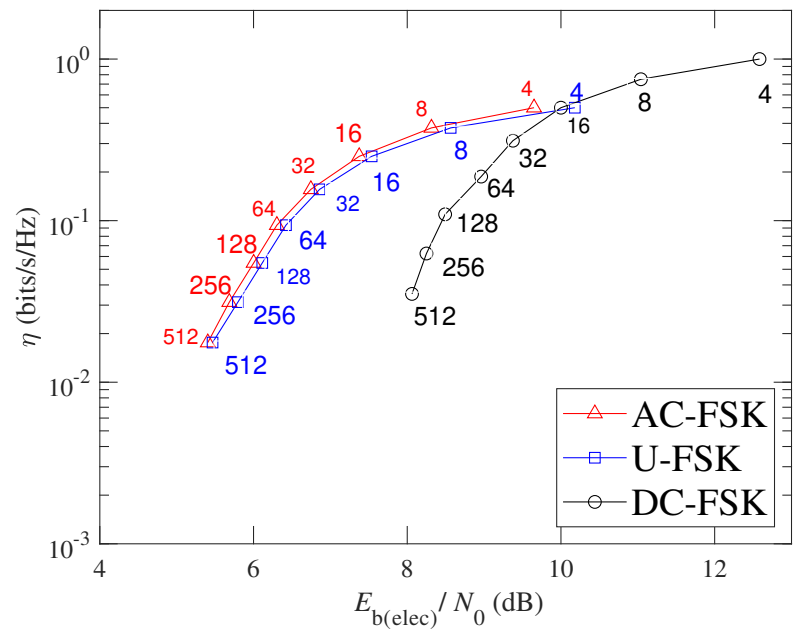

Fig. 6: Spectral Efficiency $\eta$ vs required $E_{b(\text { elec })} / N_{0}$ comparison to achieve a BER of $10^{-3}$ for $M$-ary AC-FSK, $M$-ary U-FSK and $M$-ary DC-FSK for different modulation orders using optimal receivers.

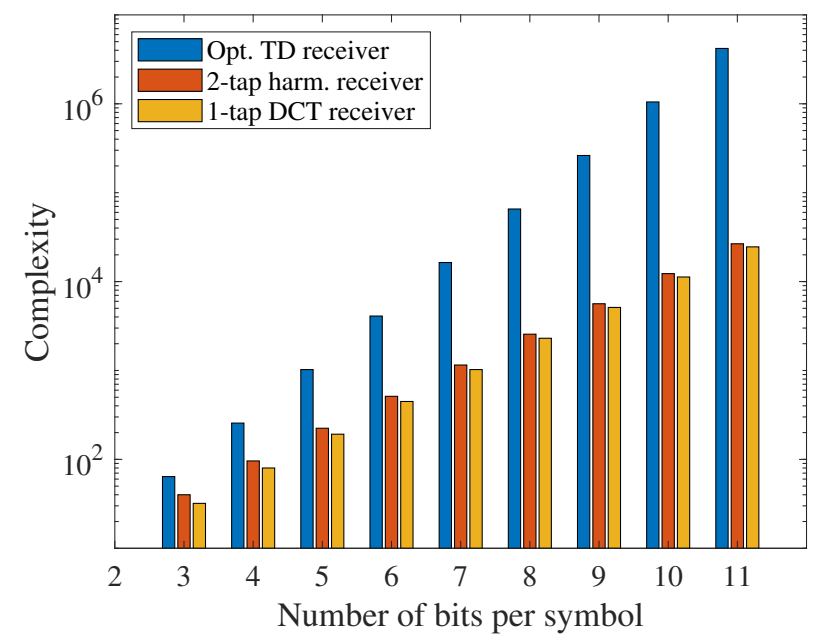

Fig. 7: Complexity comparison of optimal time-domain receiver (blue), suboptimal 1-tap DCT receiver (orange) and 2-tap harmonic receiver (red) as a function of the number of bits per symbol $\lambda=\log _{2}(M)$ for $M$-ary AC-FSK.

for $\eta=0.1 \mathrm{bit} / \mathrm{s} / \mathrm{Hz}$ that $M$-ary DC-FSK has a penalty of 2 dB relatively to $M$-ary U-FSK and $M$-ary AC-FSK.

\section{Complexity Comparison}

For $M$-ary AC-FSK, we consider only real multiplications (and not additions) for computing the complexity of the receivers because it consumes more bit operations. It is presented in [9] that $M$-order DCT requires $\mathcal{O}\left(M \log _{2}(M) / 2\right)$ real multiplications. Fig. 7 illustrates the complexity comparison of considered receivers as a function of the number of bits per symbol $\lambda=\log _{2}(M)$ for $M$-ary AC-FSK. However, the optimal ML TD receiver has better BER performance by the cost of high complexity and its complexity increases quadraticaly with $M$, while the complexity of sub-optimal and 2-tap harmonic receivers increases linearithmically with $M$. For a large $\lambda$, i.e., $\lambda \geq 7$, the complexity of 2-tap harmonic receiver is divided by a factor of 10 as compared to the optimal time-domain receiver. It can be noticed that the 2-tap harmonic receiver has slightly high complexity than sub-optimal 1-tap DCT receiver but with the benefit of better BER performance (similar BER performance than optimal time-domain receiver), or equivalently better energy efficiency.

\section{CONCLusions AND Future Work}

We have introduced a new modulation scheme called $M$ ary AC-FSK for low data rate VLC based IoE applications, and also compared its performance with state-of-the-art $M$ ary U-FSK modulation technique. $M$-ary AC-FSK modulation scheme is derived from conventional bipolar $2 M$-ary FSK, discarding the even frequency waveforms, and by clipping to zero the negative samples of odd frequency waveforms to make them compatible with IM-DD. Spectral analysis of ACFSK waveforms reveals that only 2 harmonics (leaving aside DC component) have significant amplitudes. Optimal/high complexity and suboptimal/low complexity 1-tap DCT receivers have been introduced for $M$-ary AC-FSK. As for $M$ ary U-FSK modulation, there exists an energy efficiency versus complexity trade-off for both receivers. To alleviate this tradeoff, a 2-tap harmonic receiver has been proposed. It relies on spectral properties of AC-FSK waveforms to exploit the only 2 most significant harmonics of AC-FSK waveforms to build an harmonic receiver with drastically reduced complexity: the number of multiplications becomes linearithmic with the size of the alphabet instead of being quadratic. Simulation results, considering an AWGN channel, show that $M$-ary AC-FSK with 2-tap harmonic receiver has similar energy efficiency performance as $M$-ary AC-FSK/U-FSK with optimal receiver, but with an extremely reduced complexity, comparable to those of the 1-tap DCT sub-optimal receiver.

Future works will investigate analytical BER expressions for $M$-ary AC-FSK, the impact of time-dispersive VLC channel on AC-FSK performance, and also a VLC experimental demonstration.

\section{REFERENCES}

[1] H. Haas, L. Yin, Y. Wang, and C. Chen, "What is LiFi?" Journal of Lightwave Technology, vol. 34, no. 6, pp. 1533-1544, 2015.

[2] S. Schmid, B. von Deschwanden, S. Mangold, and T. R. Gross, "Adaptive software-defined visible light communication networks," in 2017 IEEE/ACM Second International Conference on Internet-of-Things Design and Implementation (IoTDI), IEEE, 2017, pp. 109-120.

[3] A. Nuwanpriya, S.-W. Ho, J. A. Zhang, A. J. Grant, and L. Luo, "PAM-SCFDE for optical wireless communications," Journal of Lightwave Technology, vol. 33, no. 14, pp. 2938-2949, 2015.

[4] S. D. Dissanayake and J. Armstrong, "Comparison of ACOOFDM, DCO-OFDM and ADO-OFDM in IM/DD systems," Journal of lightwave technology, vol. 31, no. 7, pp. 1063-1072, 2013.

[5] Y. Roth, J.-B. Doré, L. Ros, and V. Berg, The physical layer of low power wide area networks: Strategies, information theory's limit and existing solutions, 2018. 
[6] A. W. Azim, A. Rullier, Y. Le Guennec, L. Ros, and G. Maury, "Energy efficient M-ary frequency-shift keying-based modulation techniques for visible light communication," IEEE Transactions on Cognitive Communications and Networking, vol. 5, no. 4, pp. 1244-1256, 2019.

[7] N. Fernando, Y. Hong, and E. Viterbo, "Flip-OFDM for unipolar communication systems," IEEE Transactions on Communications, vol. 60, no. 12, pp. 3726-3733, 2012.

[8] N. Ahmed, T. Natarajan, and K. R. Rao, "Discrete cosine transform," IEEE transactions on Computers, vol. 100, no. 1, pp. 90-93, 1974.

[9] J. Zhou, Y. Qiao, T. Zhang, E. Sun, M. Guo, Z. Zhang, X. Tang, and F. Xu, "FOFDM based on discrete cosine transform for intensity-modulated and direct-detected systems," Journal of lightwave technology, vol. 34, no. 16, pp. 3717-3725, 2016. 